\title{
La dinámica de sistemas
} en la enseñanza de las ecuaciones diferenciales

Raúl Serna-Laignelet

Recibido el 28 de enero de 20111 . Aprobado el 19 de abril de 2011

\section{Resumen}

El método tradicional para la solución de problemas prácticos con la ayuda de las Ecuaciones diferenciales es el desarrollo de las ecuaciones directamente de la descripción del problema. Este método requiere típicamente un proceso de prueba y error al ir de la descripción a las ecuaciones y viceversa hasta que las ecuaciones desarrolladas capturan la naturaleza del problema. En este artículo se propone un procedimiento diferente. Primero extrae una hipótesis dinámica de la descripción del problema. Paso seguido, se elaboran relaciones de causalidad cerrada o bucles. Estos bucles sirven para detectar el tipo de comportamiento o hipótesis dinámica obtenida en la primera etapa. Esta fase no es difícil ya que cada bucle genera un comportamiento dinámico específico. El último paso consiste en escribir las ecuaciones directamente de los bucles. Esta última fase es muy sencilla. Se puede resolver el sistema de ecuaciones por el método más convenga. El artículo explica el procedimiento mediante un caso, el cual describe la forma como se propaga un rumor en una población. El autor considera que el uso de este nuevo método trae varias ventajas. En primer lugar el procedimiento da mas profundidad al análisis del problema. Segundo el procedimiento es fácil de aplicar, y tercero, prepara al estudiante en áreas como la dinámica de Sistemas y el Modelaje de sistemas. La metodología fue inicialmente elaborada pensando en estudiantes de Ingeniería Industrial, Administración y Economía, pero también puede ser de utilidad a estudiantes de otras áreas de la Ingeniería, así como de las Ciencias y las Ciencias Sociales.

\section{Palabras clave}

Bucles. Causalidad Cerrada. Bucles Positivos. Bucles Negativos. Variables de Estado. Acumulación. Tasas de Cambio. Comportamiento Dinámico, Ecuación Diferencial, iThink.

\begin{abstract}
The traditional approach to solve practical problems with the aid of Differential Equations is to develop the equations directly from the verbal description of the problem. This approach typically includes trial and error procedures derived from the description of the equations, and vice versa until it is felt that the equations captures the nature of the problem. The author of this paper, proposes an alternative approach. First, he extracts a dynamic hypothesis from the description of the problem. Then, he develops a set of causal feedback loops, which in principle generate the behovior displayed by the dynamical hypothesis postulated in the first step. This phase is not difficult, since every feedback loop produces a dynamic behavior. Lastly, from the postulated set of feedback loops development of the differential equations becomes easy and possible. Solving the derived equations can be accomplished using any method chosen. This paper explains each of these steps by using the case example. The Propagation of Rumors within a Population. The author believes that this new approach to develop Differential Equations, brings several advantages. First, the approach develops a deep understanding of the problem; second, it is much simpler to apply, and third, it prepares the student in related areas of knowledge such as Systems Dynamics and Modeling. The approach was conceived primary for the use in Industrial Engineering, Business Administration and Economics, but it can also serve in other fields of Engineering, as well as, the Physical and Social Sciences.
\end{abstract}

\section{Keywords}

Feedback Loops. Closed Causality. Positive Loops. Negative Loops. State Variable. Stocks. Rate of Change. Behavior Mode. Differential Equation, iThink. 


\section{El Origen de las Ecuaciones Diferenciales}

Cualquier proceso dinámico, en cualquier entorno, representa un conjunto de variables cuyo valor cambia a medida que el tiempo trascurre. Los diferentes procesos pueden representar un sin número de patrones de comportamiento, muchos de ellos bien conocidos. Hay procesos cuyo comportamiento muestra crecimiento, hacia un límite; otros decaen gradualmente, hasta desaparecer; y muchos otros muestran oscilaciones que se repiten antes de irse amortiguando. Otros procesos muestran crecimientos sin límite, u oscilaciones cada vez mayores, hasta que el sistema propiamente dicho se destruye. Las Ecuaciones Diferenciales permiten anticipar todos esos movimientos, y son de una ayuda invaluable ya que ayuda a entender y predecir todos estos fenómenos.

Sin embargo, el proceso mediante el cual se desarrolla una ecuación diferencial no es siempre fácil de generar. Normalmente, se comienza con la narrativa de una situación y de esta se procede a elaborar directamente la ecuación diferencial, con la esperanza de que la misma represente en buen grado el fenómeno que se busca analizar.

El presente artículo presenta un método alternativo al tradicional en el desarrollo de las ecuaciones diferenciales que rigen cualquier proceso dinámico. El autor considera que el método puede perfectamente ser usado en el salón de clase y tiende a generar mucho más entusiasmo y motivación entre los alumnos que estén cursando esta materia por primera vez. Se espera que estos alumnos desarrollen el hábito y el gusto por esta disciplina y que lo mantengan después durante toda su vida profesional.

El artículo y los ejemplos que vienen han sido elaborados pensando en estudiantes de Ingeniería Industrial, Administración y otras carreras afines, pero naturalmente puede ser leído sin dificultad por cualquier estudiante de otras áreas relacionadas con la Ingeniería, las Ciencias o las Matemáticas.

La Metodología estará explicada mediante un ejemplo que se va desarrollando gradualmente. Inicia con un caso sencillo que muestra la forma como se expande un rumor dentro de una población. El sistema esta representado por un sistema no lineal de ecuaciones diferenciales e interesa principalmente, conocer una manera para encontrar esas ecuaciones. También se discute el comportamiento de ese sistema, hasta llegar a una solución, analítica de ser esta posible.
A medida que se vaya complejizando el modelo, irá apareciendo un sistema más complejo de segundo orden, también no lineal, y el cual tiene solución analítica solo en algunos casos.

\section{El Método Propuesto}

En las ciencias físicas, el desarrollo de una Ecuación deferencial que representa un proceso se efectúa en forma casi inmediata. Esto ocurre porque generalmente los procesos van definidos por leyes físicas bien conocidas y de uso común. Por ejemplo, la caída libre de un paracaidista que cae a velocidad constante. En este caso se empieza por igualar la fuerza neta de caída, masa $x$ aceleración, con la fuerza neta resultante entre el peso, la fuerza de la gravedad, $m g$ y la fuerza de resistencia del aire que se le opone, $m y$ " =mg- $k y$; ya que la caída debe ser a velocidad constante se plantea que $y "=0$ Este procedimiento, que va directamente de la narrativa del problema a las ecuaciones, es el procedimiento estándar para encontrar una ecuación diferencial en un proceso regido por leyes naturales bien definidas y conocidas.

Caso muy diferente ocurre en las ciencias sociales en donde muchas relaciones son a menudo inferencias "posibles" entre dos o más variables. Y no se conocen a total cabalidad las leyes que rigen esas relaciones. Esta situación ocurre con mucha frecuencia en problemas de Ingeniería Industrial, en Administración y en el área de la Economía. En estos casos la metodología usada para el planteamiento de una o más ecuaciones diferenciales es bien diferente. En estos casos se pueden hacer conjeturas sobre el tipo de repuesta del sistema, o bien se hace un experimento que brinde datos suficientes para establecer con algún grado de certeza la conjetura buscada.

De dicha instancia, se plantea como ejemplo la generación y propagación de un rumor en una sociedad. Cuando se trata de establecer relaciones posibles de causalidad que nos ayuden a explicar el fenómeno, se hace necesario el uso de "diagramas de influencia" o "bucles" que muestren como se relacionan o se podrían relacionar algunas de las variables del sistema para que generen la dinámica observada o la que estamos postulando.

En el caso de la propagación de un rumor, al empezar la propagación, pocos conocen el contenido de ese rumor, pero poco a poco la velocidad de propagación va aumentando, hasta que la noticia se propaga a gran velocidad dentro de la población afectada. Sin embargo, a medida que el rumor empieza a ser "vox populi" y mucha gente ya lo conoce, 
da la sensación de que la propagación del mismo se va estancando. Naturalmente hay un límite máximo de personas que llegan a conocer el rumor. El rumor no puede ser conocido por un número mayor al máximo de personas en la sociedad afectada. Así pues, el punto de partida en esta situación viene dado por una hipótesis de operación, tal como se muestra en la Figura 1.

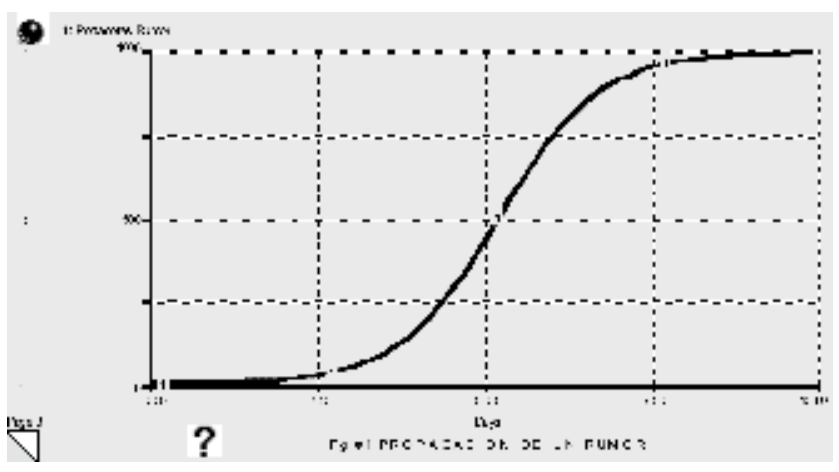

Figura 1. Propagación de un rumor. Fuente: el autor. 2011

La hipótesis de la evolución del rumor muestra un crecimiento en "S" muy frecuente en procesos de crecimiento con límite superior. En la primera fase el crecimiento es prácticamente exponencial, mientras que después, a medida que el rumor se ha expandido a la mayoría de la población, la propagación del rumor va cediendo hasta aproximarse asintóticamente al límite superior, la máxima población afectada, en este caso 1000 individuos, como se ve en la figura. La curva muestra un punto de inflexión en el momento del cambio de curvatura de crecimiento cóncavo a convexo. En este momento la Tasa de Propagación es un máximo, Tal como se muestra en la Figura 2.

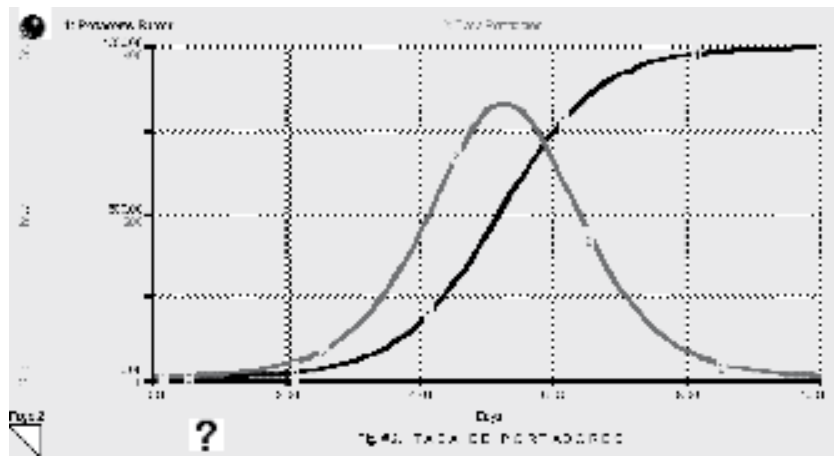

Figura 2. Tasa de portadores. Fuente: el autor. 2011

La Tasa de Propagación que era cero al empezar el proceso, crece hasta un máximo, en donde el proceso de propagación muestra expansión rápida, para luego decaer gradualmente hasta cero nuevamente, cuando ya el rumor se ha expandido a toda la población. La Tasa de Propagación representa obviamente, la primera derivada con respec- to al tiempo del número de portadores del rumor, y cuyas dimensiones son nuevos portadores de rumor por día.

\section{Metodología}

\section{Fase I. Hipótesis Dinámica.}

Las hipótesis dinámicas presentadas arriba, representan la Fase I de la Metodología que se propone al desarrollar una ecuación diferencial. De esta hipótesis se tienen que construir nuevas descripciones o narrativas que expliquen verbalmente la forma como van aumentando los Portadores del rumor y como es que al cabo de un tiempo ya el rumor no se propaga más. Esto se representa en un diagrama de influencia, que enlaza posibles causas a efectos y que tiene la particularidad de terminar con el último efecto afectando nuevamente la causa original. Estos diagramas de influencia o bucles se describen a continuación, en la Fase II.

\section{Fase II. Bucles de Causalidad.}

De la hipótesis de operación resulta evidente que la primera fase de propagación del rumor, es crecimiento de tipo claramente exponencial, y el cual resulta de un proceso que se auto-alimenta a sí mismo. En otras palabras, cuando ya hay portadores del rumor, existe la tendencia a que el rumor continúe expandiéndose a otros individuos no portadores. A medida que aumentan los portadores del rumor, aumenta la Tasa de Propagación, lo cual hace aumentar aún más el número de Portadores. Es lo que se llama un Bucle de re-fuerzo positivo, y caracteriza siempre un proceso de crecimiento exponencial como el se ha venido analizando (Anderson \& Johnson, 1997), (Sterman, 2001). Sin embargo como ya se observó, al revisar las hipótesis de operación, este proceso se altera a medida que la población portadora aumenta hacia el total de la población. Ver Figura 3.

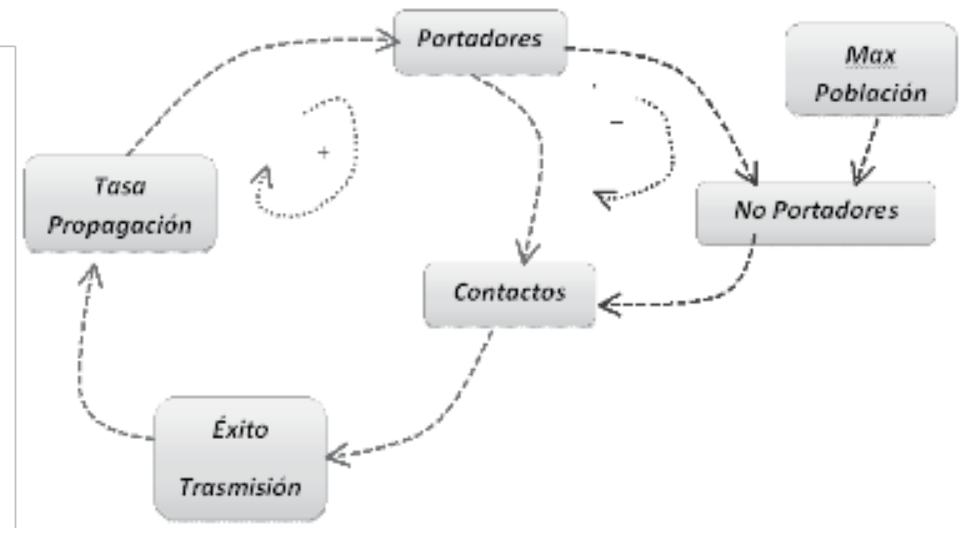

Figura 3. Bucles de casualidad. Fuente: el autor. 2011 
En la Figura 3, se puede observar que a medida que aumenta la población de Portadores, se va agotando la de los No Portadores, en tal forma que nuevos éxitos en la transmisión del rumor se van haciendo cada vez más difíciles de lograr.

Se puede entonces, hacer la hipótesis en que la Tasa de Propagación depende en últimas de dos factores, uno que depende del número de Propagadores y el otro del número de No Propagadores que permanezcan en el sistema. Es decir,

$$
\begin{gathered}
\text { Tasa de Propagación }=G(\text { Propagadores, } \\
\text { No Propagadores) }
\end{gathered}
$$

\section{Fase III. La Ecuación Diferencial}

Los bucles en la Figura 3 muestran con mayor claridad la forma como opera el proceso. Un bucle marcado "+" es de re-fuerzo positivo, y causa crecimiento (exponencial); el otro marcado "-", es un bucle negativo y causa atenuación en el efecto de crecimiento generado por el primer bucle.

Antes se dijo que la Tasa de Propagación dependía de dos factores, uno que dependía de los "Propagadores del Rumor" con un efecto positivo, que hace aumentar la Tasa de Propagación; el otro factor, dependía de los "No Propagadores", con un efecto opuesto que hace disminuir la Tasa de Propagación. Puesto que el total de la población está dado por la suma de los Portadores con los No Portadores, la Tasa de Propagación viene a ser una función de los Portadores, o sea,

No Portadores $=$ Max. Portadores - Portadores

$$
\begin{gathered}
\qquad N P=M-P \\
\text { Tasa de Propagación }=g(P, M-P)=f(P)
\end{gathered}
$$

Esta función, creciente en la fase inicial de la propagación, debe llegar a un máximo y posteriormente decaer hasta llegar a cero cuando ya se haya completado toda la propagación del rumor, tal como aparece en la Figura 4. Una función que cumpla esta condición y que puede servir para representar este proceso es una parábola, tal como se presenta a continuación,

\section{Sistema no lineal de primer orden}

$$
\frac{d}{d l} P=k P(M-P)
$$

Siendo: $\mathrm{P}=$ Portadores del Rumor, $\mathrm{M}=$ Población Total y $\mathrm{k}=$ Constante de Propagación
Aquí la Tasa de Propagación se ha representado por la primera derivada con respecto al tiempo de los Portadores, y los Portadores son una función del tiempo $P(t)$, La tasa de Propagación $P^{\prime}$ aparece como una función de $P$ como se observa en la Figura 4.

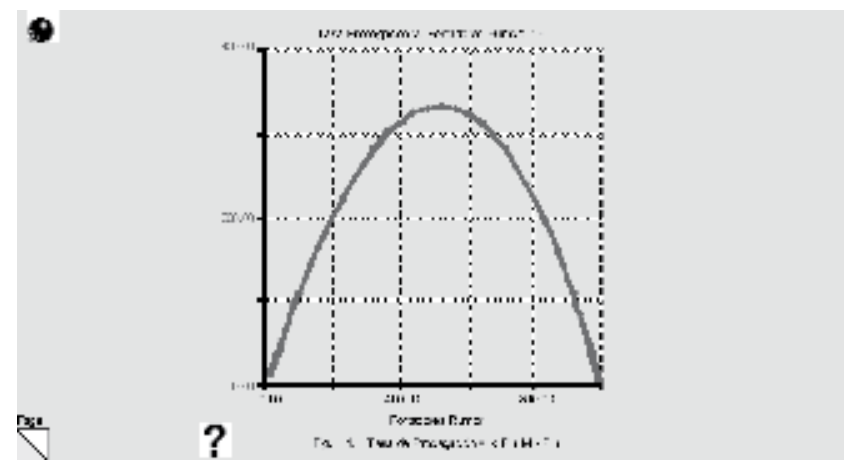

Figura 4. Tasa de Propagación. Fuente: el autor. 2011

En esta representación, k es una constante que combina la fracción de éxitos en la transmisión del rumor y la velocidad a la cual se propaga el mismo, $M$ es el máximo de Población susceptible de ser portadora y $\mathrm{P}$ es el número de Portadores en un momento determinado.

La solución de esta ecuación diferencial se determina mediante el método de separación de variables. Su solución es la función Logística.

$$
P(t)-\frac{M P_{0} e^{i t i t i}}{M-P_{0} e^{\frac{1}{n} \cdot m t}}
$$

\section{Donde $P_{0}$ representa la cantidad inicial de portadores}

El sistema que se describe, admite otras representaciones diferentes, pero similares y en últimas representan la misma Ecuación Diferencial. En el presente ejemplo, se puede representar al conjunto de los No Propagadores como una variable de estado que vaya disminuyendo a medida que el tiempo va transcurriendo. Como se muestra en la Figura 5, se tienen nuevamente dos bucles, uno que hace disminuir a la cantidad de No Portadores, a medida que la Tasa de Propagación aumenta, y otro que hace aumentar a la cantidad de Portadores a medida que la misma Tasa de Propagación aumenta. Al primer bucle se le denomina un Bucle Negativo porque su efecto es oponerse al aumento en la variable, No Portadores. El otro Bucle es Positivo o de refuerzo por que hace aumentar a la variable Portadores. Ver Figura 5.

Este sistema de bucles contiene las mismas dos variables de estado del modelo anterior, una representada por los No Portadores, NP, la otra por los Portado- 
res, P. El sistema esta regido por el siguiente par de ecuaciones,

\section{Sistema No lineal de Segundo Orden}

$$
\begin{aligned}
& \frac{d}{d t} P=k P \cdot N P \\
& \frac{d}{d t} N P=-k P \cdot N P \\
& M=N P+P
\end{aligned}
$$

Siendo: $P=$ Portadores del Rumor, $M=$ Población Total, $k=$ Constante de Propagación y $N P=$ No Portadores del Rumor

La Figura 5 muestra el modelo con la nueva representación, en donde aparece un bucle que contiene a los No Portadores y otro que contiene a los Portadores. Los dos bucles están unidos por la Tasa de Propagación, que afecta a las dos variables de estado. Esta nueva representación, en la Figura 5, trae algunas ventajas ya que ofrece más flexibilidad al incorporar otros elementos al modelo, como se verá posteriormente. Este nuevo sistema de bucles es enteramente equivalente al que vimos anteriormente, y cuya solución es por supuesto la función logística. La solución se muestra en la Figura 6.

Al final de diez días toda la población de No Portadores ha pasado a formar parte de la población Portadora, En el modelo de la solución en la Figura 6 , se han usado los siguientes valores para los parámetros: $\mathrm{M}=1000$ (Máx. Población) y $\mathrm{k}=$ 0.001 como constante de propagación. El valor inicial de Portadores es de 10 individuos, y al empezar se asumió una población de No Portadores de 1000 individuos.

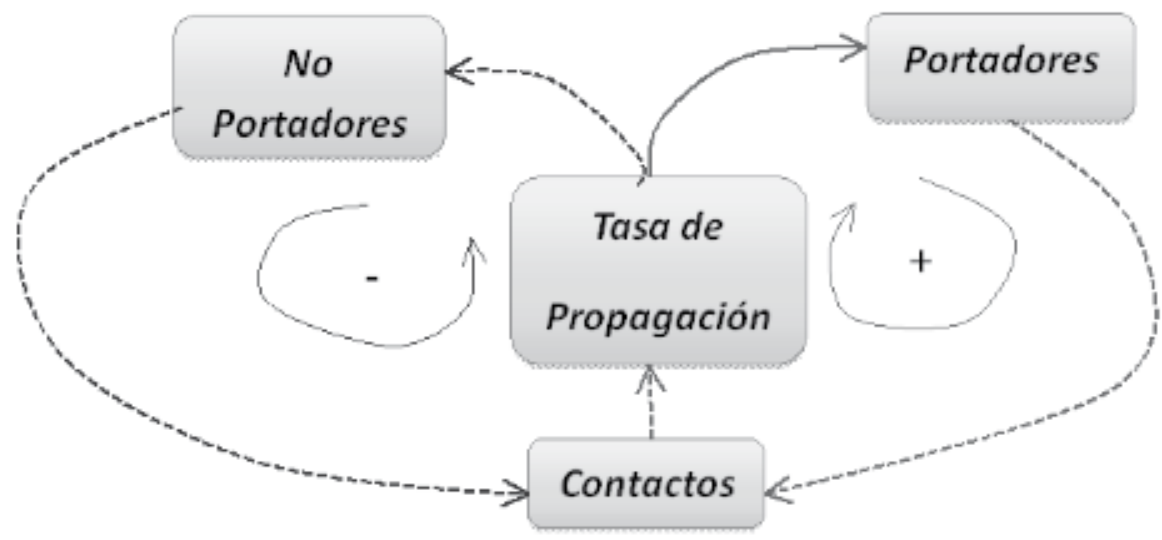

Figura 5. Fuente: el autor. 2011

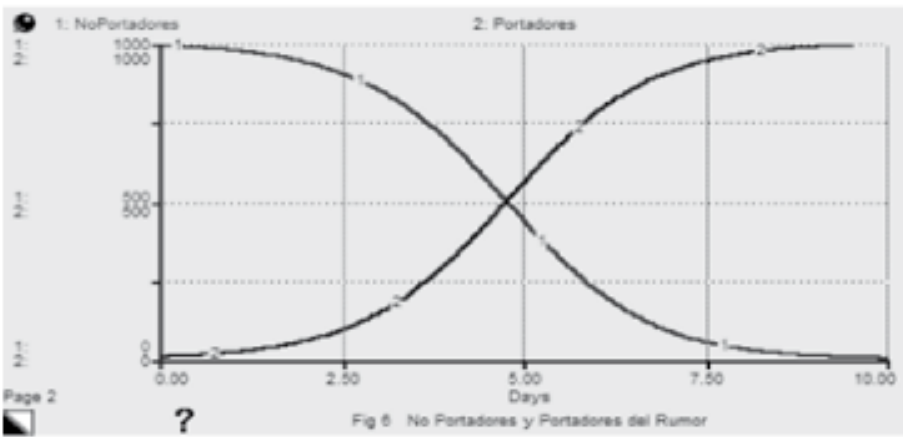

Figura 6. No portadores y Portadores del rumor. Fuente: el autor. 2011

\section{Fase IV. Modelos. Tasas de Cambio y Acumulaciones.}

Una de las ventajas de usar la representación de bucles en la Figura 5, es que permite estudiar otros casos más complejos que requieren variar la ecuación diferencial. Por ejemplo, se puede analizar la situación en la cual hay un flujo de nuevas personas entrando y otro flujo de personas saliendo del sistema, o inclusive analizar el caso en el cual algunos portadores de rumor deciden permanecer en el sistema pero no continuar divulgando el rumor, quizás porque ya no creen la información o bien porque no lo desean.

En la Figura 7, se representa el modelo agregándole nuevamente un par de bucles, uno de entradas de nuevos individuos, No Portadores, al sistema, el otro mostrando salidas de Portadores del sistema. Nótese que el bucle de entradas se ha visualizado como un bucle positivo de refuerzo ya que indica que a más número de No Portadores, mayor el número de individuos que entran al sistema. Este bucle tiende a producir crecimiento en la población total. Por el contrario, el bucle de salida, tiene el efecto contrario. A más Portadores de rumor, existe una cantidad de individuos que desean salir del sistema. Este segundo bucle de salidas podría, por supuesto, haber sido modelado en otra forma. Lo importante aquí es que este bucle es Negativo, y tiende a atenuar o disminuir la población de Portadores y de la Población en general.

Ahora se tienen tres Tasas de Cambio. La Tasa de Entradas que afecta a los No Portadores, la Tasa de Salidas, que afecta a los Portadores, y la ya conocida Tasa de Propagación. 


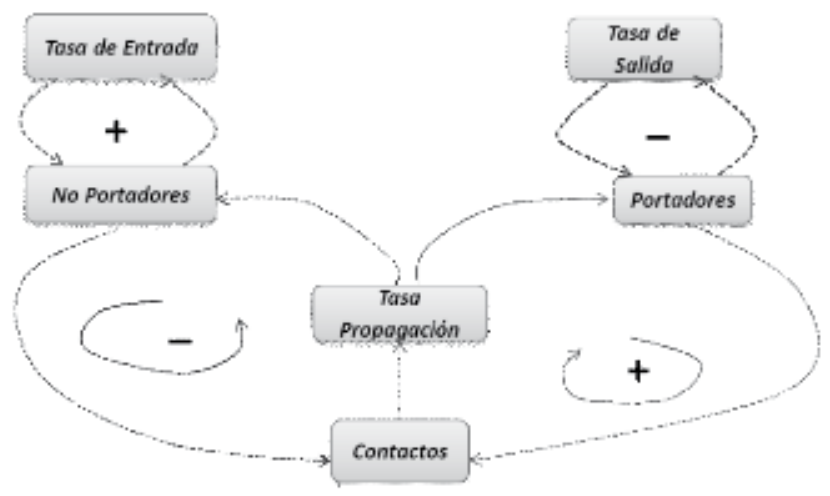

Figura 7. Fuente: el autor. 2011

\section{Acumulaciones y Variables de Estado}

Las variables No Portadores y Portadores, son variables de estado del sistema. Representan grupos de individuos, y por eso se distinguen denominándolos acumulaciones o variables de nivel o variables de estado. Estas acumulaciones reciben flujos de individuos por día que se van uniendo al sistema o van saliendo del mismo, y es por eso que a esos flujos se les conoce como tasas de cambio. Por ejemplo, a la acumulación No Portadores le llega un flujo de nuevas entradas al sistema, y de esa misma acumulación sale un flujo, la tasa de conversión de No Portadores a Portadores o Tasa de Propagación. Figura 8.

Nótese que las variables de estado o acumulación de los No Portadores y de los Portadores, los cuadrados en la Figura 8, vienen dadas por las siguientes relaciones:

\section{Ecuaciones de las Variables de Estado Portadores y No Portadores}

No portadores $(t+d t)=$ No portadores $(t)+(d t) \quad$ (taza de Entrada - Tasa de Propagación

Portadores $(t+d t)=$ Portadores $(t)+(d t)$ (Tasa de Propagación - Tasa de Salida

Siendo $(t)=$ Tiempo y $d t=$ Lapso de tiempo $\longrightarrow 0$

Es decir, una variable de estado en un tiempo $t+d t$ es igual a esa variable en el tiempo $t$ más los flujos que entran menos los que salen durante el lapso de tiempo $d t$ Esta relación es enteramente similar a decir que la Tasa Neta de Cambio en una variable de estado, cuando el lapso de tiempo $d t$ es muy pequeño, es igual a la suma de las tasas netas de cambio que afectan a esa variable de estado, Para el caso se tiene,

$\frac{d}{d t} \mathrm{~s}^{\prime}(t)=$ Tasa de Entrada - Tasa de Propagación

$\frac{d}{d t} f^{\prime}(t)=$ Toso de soldo - Toso de Propagoción
El diagrama de la figura 8, representa el "esqueleto" del sistema y representa un paso posterior a los diagramas de bucle que ya se han visto. Este tipo de diagrama conocido como el diagrama Forrester (Forrester, 1961), (Sterman, 2001), representa en forma explícita cada una de las relaciones involucradas en el sistema.

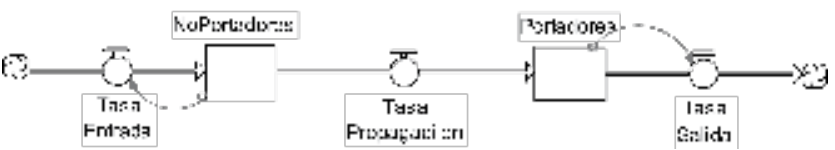

Figura 8. Esqueleto del Modelo Acumulaciones y Tasas de Cambio. Fuente: el autor. 2011

En la Figura 9 se ha completado el Esqueleto del Modelo con los Flujos de información y con los Bucles que operan del sistema.

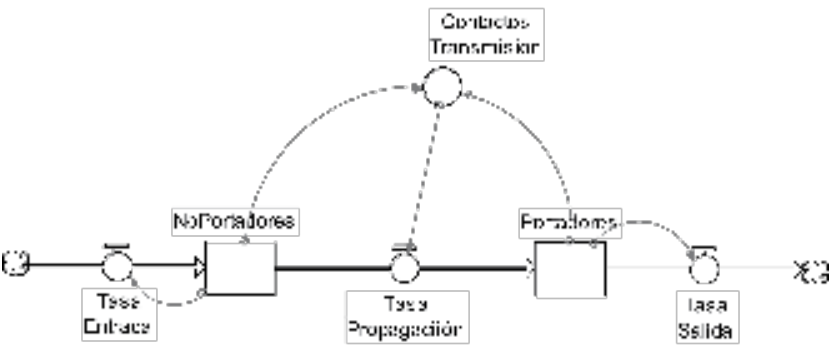

Figura 9. Esqueleto del Modelo Acumulaciones y Tasas de Cambio con Bucles de Operación. Fuente: el autor. 2011

En este caso, con bucles de entrada y de salida, el sistema de Ecuaciones es más complejo que en los casos que ya se han visto, como se puede observar en las siguientes ecuaciones:

\section{Sistema No Lineal de Segundo orden con Entradas y Salidas}

$\frac{d}{d} P=-\beta P+=O \cdot N P+k P \cdot \lambda P$

$\frac{d}{d t} H P^{2}=O \cdot l^{\prime}+\alpha P^{2}-h P^{3} \cdot W P^{\prime}$

Siendo: $P=$ Portadores del Rumor, $k=$ Constante de Propagación, $N P=$ No Portadores del Rumor, $\alpha=$ Tasa porcentual de entradas y $\beta=$ Tasa porcentual de salidas.

Las constantes alfa y beta en estas ecuaciones, son respectivamente las tasas porcentuales de entrada y de salida. La otra constante k es la tasa porcentual de propagación como ya se vio con anterioridad.

Este par de ecuaciones diferenciales representan un sistema no lineal, de segundo orden, cuya solución analíitica para $P(t)$ y $N P(t)$ no es posible. En este caso, sin embargo, las ecuaciones admiten un tipo de solución mediante el método de separación de variables. Esta solución produce una variable de estado 
en función de la otra, $N P=f(p)$ y se llama la "órbita" del sistema. Una solución de este tipo es muy útil, aun en el caso en que se haya podido encontrar soluciones para $P$ y NP en función del tiempo. Usando la técnica de separación de variables se obtiene:

$$
\begin{aligned}
& \frac{d}{d t} P=P(-\beta+k N P) \\
& d N P=N P^{\prime}\left(\alpha-k l^{\prime}\right) \\
& d t \\
& \frac{d l^{\prime}}{d N P}=\frac{f^{\prime}\left(-\beta-k P^{\prime}\right)}{N P(\alpha-k P)} \\
& \int \frac{(\alpha-k P)}{P} d P=\int \frac{(-\beta+h N P)}{N P} d N P+c \\
& \log \left[P^{p} N P^{\beta}\right]-k\left(P^{\prime}+N P^{\prime}\right)=c
\end{aligned}
$$

Esta última relación entre NP y $P$ es la órbita solución del sistema. De la forma de esta órbita, y usando algunos conceptos de la Teoría Cualitativa de las Ecuaciones Diferenciales (Jordan \& Smith, 2007), (Lynch, 2007), (Strogatz, 1994), se sabe que para cada valor de la constante de integración c el mapa formado entre NP y P está cerrado. Cuando esto ocurre la solución en función del tiempo muestra oscilaciones.

Estas oscilaciones se pueden observar resolviendo el sistema mediante cualquiera de los métodos numéricos conocidos. El software iThink usa el método de Euler o uno de los métodos Runge-Kuta para mayor precisión. El resultado, usando un Runge Kuta de cuarto orden se muestra en las figuras 10 (a y b).

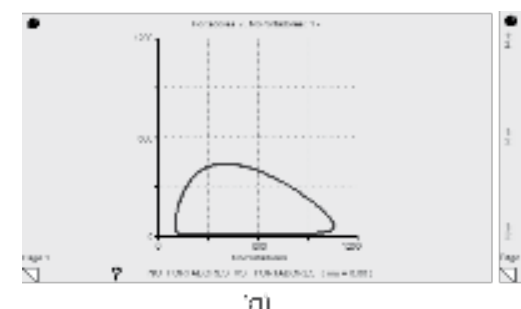

(i)

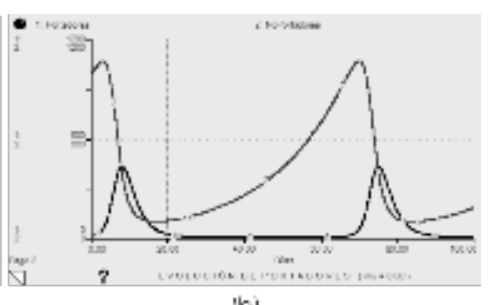

$|b|$
Figura 10. Fuente: el autor. 2011

En la figura superior izquierda aparece el "Retrato de las Fases", u órbita, que muestra los Portadores vs. No Portadores, La órbita es una curva cerrada, algo característico cuando el sistema produce oscilaciones sostenidas, como las que se ven en el cuadro a la derecha. Para obtener estos resultados se usaron los siguientes valores.

\section{Valores de los parámetros del Sistema}

$\alpha=$ Tasa porcentual de entrada $=0.05$

$\beta=$ Tasa porcentual de salida $=0.40$

$\mathrm{k}=$ tasa porcentual de propagación $=0.001$
Valores iniciales de las Variables de Estado

$N P=$ No Propagadores $=1000$ Personas

$P=$ Portadores $=10$ Personas

La Figura 11, muestra el comportamiento durante los primeros 15 días de propagación del rumor. De esta figura no es nada evidente la existencia de un movimiento oscilatorio en el sistema. Este movimiento oscilatorio ha sido causado al agregar los bucles de entrada y de salida. Como se ve, los "bucles" en general, tienden a modificar la forma como un sistema se comporta. Un bucle es un ente poderoso ya que genera cambios importantes en los sistemas.

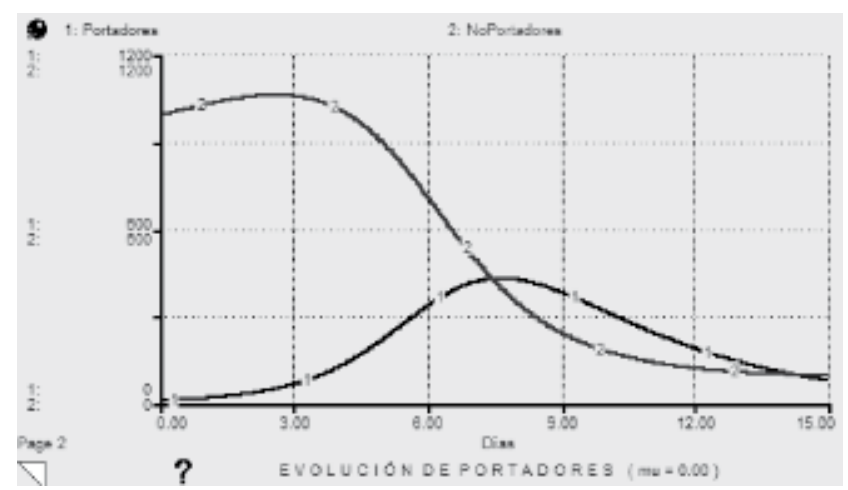

Figura 11. Evolución de Portadores. Fuente: el autor. 2011

En el último caso que se va a presentar, se incluye un flujo de retorno de Portadores a No Portadores. Representa a Portadores que quieren permanecer en el sistema, pero que no desean continuar propagando el rumor. Son individuos que regresan al conglomerado No Portadores y pueden ser susceptibles a volver a ser Portadores después de un tiempo. No todos regresan, solo una fracción $\mu$ de ellos lo hace $(\mu=0.40)$ En este caso las ecuaciones de estado para los Portadores y los No Portadores varían en la siguiente forma:

$$
\begin{aligned}
& \text { Sistema No Lineal de segundo Orden } \\
& \frac{d}{d f} \rho=-(\beta-\mu) r^{\prime}+O \cdot N P^{\prime}+k f^{\prime} \cdot N \\
& \frac{d}{d t} k P=\mu p^{2}+\alpha N W^{2} \quad k p^{\prime} \cdot h P
\end{aligned}
$$

Siendo: $P=$ Portadores del Rumor, $k=$ Constante de Propagación, $N P=$ No Portadores del Rumor, $\alpha=$ Tasa porcentual de entradas, $\beta=$ Tasa porcentual de salidas y $\mu=$ Tasa porcentual de regreso.

A diferencia del caso inmediatamente anterior, en estas ecuaciones ya no es posible encontrar una solución analítica para la órbita. Tampoco se conoce cual es la solución analítica para las variables de estado en función del tiempo, $P(t)$ y $N P(t)$. En el caso anterior, la órbita analítica encontrada llegó inclusive 
a indicar que el sistema iría a mostrar oscilaciones, como efectivamente se comprueba después al efectuar el cálculo numérico. Ahora se requiere usar el cálculo numérico ofrecido por el paquete iThink para encontrar el comportamiento del sistema, el cual resulta también del tipo oscilatorio.

El comportamiento del sistema, indicado por la solución obtenida en esta forma, muestra que la población de No Portadores tiende a aumentar inicialmente, hasta un valor después del cual decae, permitiendo además el crecimiento de la población de Portadores como se ve en la Figura 12.

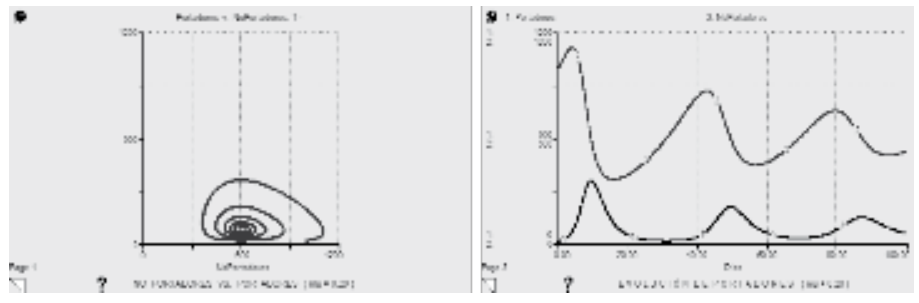

Figura 12. Fuente: el autor. 2011

El Retrato de las Fases, ( $P$ vs. $N P$ ) en espiral, que converge hacía un foco indica movimiento oscilatorio con amplitud decreciente, lo que también sugiere que el sistema es estable. Este movimiento oscilatorio tiene un periodo de unos 30 a 32 días aproximadamente. Los Portadores aumentan, llegan a un pico a los 10 días aproximadamente, y después tienden a desaparecer, para luego volver a renacer alrededor de 20 días más tarde con fuerza renovada. La anterior, es una conclusión mas bien inesperada en este sistema, pero no resulta muy sorprendente si su análisis se efectúa a la luz de los bucles y de las ecuaciones no lineales como las que se han estudiado en este artículo.

El sistema completo, mostrando el nuevo flujo de Regreso de Portadores a No Portadores aparece a continuación, en la Figura 13.

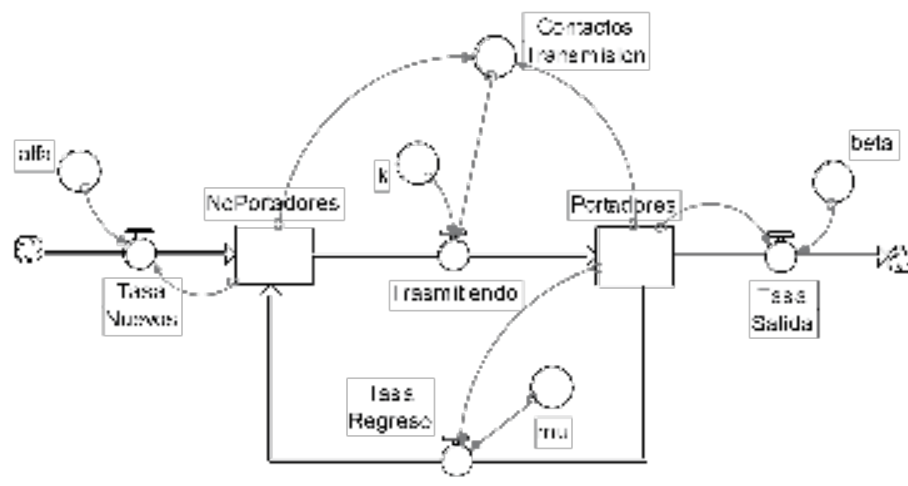

Figura 13. Sistema Completo. Fuente: el autor. 2011

Como se puede apreciar en la Figura 13, este modelo resulta más interesante para los estudiantes que la simple presentación de las ecuaciones diferenciales que rigen la Propagación del Rumor. En particular los bucles involucrados en el diagrama representan con claridad cada uno de los mecanismos que generan la dinámica del sistema. Además, el diagrama contiene cada relación postulada al construir la hipótesis de operación, y es muy fácil pasar de esta representación a la representación en las ecuaciones diferenciales o hacerlo al contrario, y de las ecuaciones diferenciales obtener un diagrama análogo al presentado. Estas son ventajas que trae la metodología que se propone en el estudio de las Ecuaciones Diferenciales.

\section{Aplicaciones y Extensiones}

Un sistema como el anterior, regido por ecuaciones diferenciales, normalmente tiene aplicaciones a otros sistemas en otros entornos y de apariencia muy diferentes. Solo basta con que la estructura de los "bucles" sea similar entre un sistema y otro para establecer algunas similitudes entre un sistema y otro. No es de sorprender ya que muchas veces hay similitudes entre ecuaciones diferenciales que representan sistemas bastante disímiles. Se puede recordar que un circuito eléctrico RLC tiene un comportamiento enteramente similar al de una Masa suspendida de un Resorte con Amortiguamiento. Estos dos sistemas, al representarlos con sus "bucles" resultan, por supuesto, con bucles muy análogos. Vale la pena tener presente, que cada "bucle" siempre representa a una ecuación diferencial y viceversa.

En el ejemplo de la Propagación del Rumor, resulta casi evidente que este sistema y su esqueleto correspondiente, pueden ser fácilmente modificados para que representen sistemas diversos, o sistemas análogos, como se les llama. Algunos de los cuales se enumeran a continuación

1. Propagación de una epidemia.

2. Un sistema de propaganda para promover un producto.

3. La captación de adeptos a una idea, a un partido, o a una religión.

4. Clínicas de Ventas, Entrenamiento a Vendedores

5. Captación de nuevos clientes en un mercado competitivo

6. Recuperación de drogadictos

Naturalmente cada uno de estas situaciones tendrá además del esqueleto general, sus propias características típicas del sistema estudiado. Estas deben naturalmente ser incorporadas en el modelo, ya sea en forma de bucles y en forma de parámetros especiales a ese sistema. En general la incorporación 
de nuevos bucles, o su eliminación, tiende a generar cambios importantes en el modo de comportamiento del sistema. El análisis es sin embargo similar al que se ha presentado en el presente artículo.

\section{Conclusiones}

Este documento presenta una manera diferente de desarrollar y analizar un sistema dinámico de Ecuaciones Diferenciales. Usa conceptos tomados de la Dinámica de Sistemas. Principalmente toma el concepto de los "Bucles" en esta disciplina. Estos bucles son estructuras retro-alimentadoras que generan dinámica, y sirven para representar cualquier descripción o narrativa de un problema. El artículo es sui-generis en la manera de pasar de las narrativas de un problema al planteo de los "bucles" y de allí al planteo de la Ecuación o Ecuaciones diferenciales involucradas en una situación dinámica.

La Metodología propuesta, hace énfasis en la relación directa que existe entre una ecuación diferencial y un bucle y entre varios bucles ya estén acoplados o no y un sistema de ecuaciones diferenciales, sean ellas ecuaciones lineales o no lineales.

El método, evita el complejo camino de pasar directamente de las narrativas a la ecuación diferencial, paso que muchas veces resulta complicado y de difícil retención, y que tiende a alejar al estudiante de esta área tan importante del conocimiento. Además de lo anterior, se puede considerar como el mayor beneficio del método, al hecho que el mismo involucra al estudiante desde el principio en la construcción de bucles de operación, los cuales serán posteriormente la piedra fundamental para sus estudios en las áreas de Sistemas Dinámicos, en el Modelaje de Sistemas y en la Simulación de los mismos. Estas son herramientas centrales para lograr mejoras en los sistemas en los que el futuro profesional intervenga. Se espera que el estudiante que incorpore esta metodología desde el principio de su carrera, pueda obtener con mayor celeridad y éxito mejoras en el comportamiento de sistemas que esté diseñando o con los que esté trabajando.

\section{Recomendaciones}

- Se recomienda continuar con el estudio analítico o simulado de algunos de los sistemas análogos indicados arriba o de otros posibles usando la metodología descrita en este artículo, a fin de incorporar el concepto de los bucles en el desarrollo y uso de las ecuaciones diferenciales y que rigen cualquier sistema.

- Sería recomendable incorporar la metodología descrita en este artículo al pensum de los cursos de Ecuaciones Diferenciales y tomar las observaciones del caso con el fin de evaluar la aceptación y los resultados de la metodología entre los futuros profesionales.

\section{Referencias}

[1] Anderson, V. \& Johnson, L. (1997), System Thinking Basics: From Concepts to Causal Loops, Cambridge, MA: Pegasus Communications, Inc.

[2] Forrester, Jay W. (1961), Industrial Dynamics, MIT Press, Republished in 1999 by Pegasus Communications.

[3] Jordan, D. W. \& Smith, P. (2007), NonLinear Ordinary Differential Equations, An introduction for scientists and engineers, Fourth Edition, New York, Oxford University Press.

[4] Lynch, S (2007), Dynamical Systems With Applications Using Mathematica, Boston, BirkHauser.

[5] Serna-Laignelet, R. (1976), World Coffee Dynamics, Ciclos Endógenos en la Industria del Café, Unplublished Doctoral Thesis, Georgia Institute of Technology (Ga. Tech.).

[6] Sterman, J. D. (2000), Business Dynamics: Systems Thinking and Modeling for a Complex World, New York, Irwin/McGraw Hill.

[7] Strogatz, S. H. (1994), Nonlinear Dynamics and Chaos, Massachusetts, Addison-Wesley Publishing Company.

Raúl Serna-Laignelet. Ingeniero Industrial. Ph.D. y MS en Ingeniería Industrial en el Georgia Institute of Technology. Ingeniero Electricista Universidad Industrial de Santander (UIS). Más de 30 años de experiencia en compañías Internacionales en las áreas de Logística, Distribución, Mercadeo y Ventas. Profesor de Matemáticas Universidad de los Andes. Áreas de Interés: Dinámica de Sistemas, Administración, Investigación de Operaciones, Logística, y Ecuaciones Diferenciales. Curso en Dinámica de Sistemas a profesores de Ingeniería en La Corporación Universitaria Minuto de Dios (UNIMINUTO), rsernal@aol.com 